\title{
IMPACT OF THE COVID-19 PANDEMIC ON LIFESTYLE AND ANXIETY LEVELS IN WOMEN WITH BREAST CANCER
}

Alice Aparecida Rodrigues Ferreira Francisco', Jader Brito Ramos da Silva', Lais Moriyama Pereira Lima', Karen W. Wonders ${ }^{1}$, Otavio Augusto Soares Machado ${ }^{1}$

'Maple Tree Cancer Alliance - Sorocaba (SP), Brazil.

Introduction: The COVID-19 pandemic, which led to an attempt to reduce infection rates, also led to radical changes in our daily lives and forced the world population to isolation and distance. As result, our lifestyle habits and mood have also changed. Whether due to the fear of performing physical activity during treatment, or the lack of information regarding the benefits associated with it, the number of sedentary cancer patients remains high. With the pandemic, the challenge of introducing and maintaining the practice of physical activity during cancer treatment as a "standard of care" became even greater. Objectives: To assess changes in physical activity levels, time spent sitting and self-reported levels of anxiety in women diagnosed with breast cancer, in the face of the COVID19 pandemic. Methods: An online questionnaire was applied to cancer patients, evaluating 57 women diagnosed with breast cancer, aged between 20 and 75 years. The questionnaire was sent via text message and on spontaneous demand on social networks. Results: As main results, we found out that 46 women (80.7\%) were practicing regular physical exercises before the pandemic, while 40 (70.1\%) of them managed to maintain this habit. The averages of weekly time (minutes) spent in physical exercise, subjective effort intensity, time (hours/day) spent watching TV, and anxiety level before and during the pandemic were $90.5 \mathrm{vs.} 69.4$ ( $\mathrm{p}<0.03)$; 2.3 vs. $1.9 ; 2.3$ vs. 3.2 ( $\mathrm{p}<0.007) ; 2.6$ vs. 3.3 ( $\mathrm{p}<0.003$ ). Regarding the percentage analyses, it was noted that even in the pre-pandemic period, only $24.5 \%$ of the sample performed more than 150 minutes of weekly physical activity, as recommended by the World Health Organization (WHO). As for the level of anxiety, when comparing the number of women who rated themselves between very anxious and exaggeratedly anxious, an increase of $110.4 \%$ was noted during the pandemic. Conclusions: Even though exercise protocols for cancer patients are well established, we observed in the present study that most patients do not meet the minimum necessary WHO recommendations. Furthermore, the increase in hours spent watching television may be a factor that contributed to increase the level of anxiety, decreasing the quality of life of these participants. 